\title{
Aerobic bacteria associated with symptomatic gallstone disease and their antimicrobial susceptibility
}

PRL Gomes ${ }^{1}$, SSN Fernando ${ }^{1}$, DD Weerasekara ${ }^{2}$, VGNS Velathanthiri ${ }^{1}$, MSM Rizny ${ }^{3}$, MM Weerasekara $^{1}$, Rohan Mahendra ${ }^{1}$

${ }^{1}$ Department of Microbiology, ${ }^{2}$ Department of Surgery, ${ }^{3}$ Department of Anatomy

Faculty of Medical Sciences, University of Sri Jayawardenapura, Nugegoda.

\begin{abstract}
Introduction: Gallstone disease is a major health problem throughout the world. Apart from surgery prompt administration of appropriate antibiotics to control the biliary tract infection is important.
\end{abstract}

Objective: Illustrate the bacteriology of gallstone disease.

Design: The bile stones and bile of patients with symptomatic gallbladder disease, who underwent cholecystectomy, over a period of one year, were cultured aerobically in blood agar and MacConkey agar. Enrichment was not done, as it was not indicated in previous studies. The isolates were identified according to standard microbiological procedures and were tested for their sensitivity pattern.

Settings: Department of Microbiology, University of Sri Jayawardenapura and Colombo South Teaching Hospital, Kalubowila, Sri Lanka.

Results: Twenty percent of the patients were positive for bacteria in either bile or stones. Escherichia coli (40\%), Klebsiella spp (35\%) and Enterobacter spp (20\%) were the commonest isolates. Ninety one percent of all isolates were Coliforms.

Conclusions: We recommend obtaining of cultures of bile and gallstones at the time of cholecystectomy so that appropriate antibiotics can be administered in the event of a positive culture 
to prevent serious complications like gram negative septicaemia. According to our results, co-amoxiclav in combination with an aminoglycoside for aerobic bacteria along with metronidazole to cover anaerobic bacteria is an empirical therapy that can be used before the results of bacteriological cultures. Cefotaxime or imipenem can be used for aerobic bacteria as an alternative treatment.

\section{Introduction}

Gallbladder disease is a health problem throughout the world.

The commonest reason being gallstones; accounting for over $90 \%$ of the cases [1].

Human bile is normally sterile [2,3]. However, in biliary tract obstruction, bacteria may gain access to the biliary tract through either the papilla of Vater or the portal circulation and subsequently lead to biliary tract infection [4]. Systemic bacteraemia may also occur in severe cases [5]. Without adequate treatment infections can lead to severe complications and death [6,7].

Surgical treatment including decompression of biliary obstruction and removal of stones is considered to be the gold standard $[8,9]$. However, prompt administration of appropriate antibiotics to control the biliary tract infection is also important $[7,10]$.

In recent years, development of resistant strains and lower susceptibility of anaerobes and gram negative rods have made antimicrobial therapy difficult and complicated [11]. The importance of obtaining cultures of the bile at the time of cholecystectomy lies in the fact that appropriate antibiotics can be administered in the event of positive culture to prevent serious complications like gram negative septicaemia [12].

Studies show $10 \%$ to $15 \%$ prevalence of gallstones in USA [13]. Scandinavian populations are found to be at high risk, with about $50 \%$ of individuals developing gallstones by the age of 50 years [14].

Studies of infections of biliary tract are sparse in Sri Lanka. There is no published data on the aetiology and the choice of antibiotics.
Conservative and prophylactic treatment is based on best guess basis. Therefore we conducted this study in an attempt to illustrate the bacteriology of gallstone disease and provide information for antibiotic choice.

\section{Method}

Project was conducted from August 2004 to August 2005. One hundred patients diagnosed with symptomatic gallstone disease who underwent cholecystectomy at Colombo South Teaching Hospital, were subjects of this prospective study. The bile and gallstones were collected to a sterile screw capped bottle after informed consent from the patient. The demographics of the patients were collected through a questionnaire.

A sample of bile and crushed gallstones were inoculated to blood and McConkey plates. After overnight incubation at $37{ }^{\circ} \mathrm{C}$, the isolates were identified according to standard microbiological procedures. An antimicrobial sensitivity test was performed (John Stroke's method) on the isolates to determine the sensitivity pattern of these isolates.

\section{Results}

Study sample was 100 . There were 83 females. The number of cases in different age groups is shown in table-1. Twenty patients were positive for bacteria in either bile or stones (both stone and bile -12 , bile only -4 , stone only -4 ). Culture positivity was 39\% (13/36) among patients over 50 years of age compared to $11 \%$ (7/64) among patients aged fifty or less.

Table 1 - Symptomatic gallstone disease in different group ages

\begin{tabular}{|l|c|c|}
\hline Age group & No. of cases & $\begin{array}{c}\text { No. of culture } \\
\text { positives }\end{array}$ \\
\hline$<20$ & 1 & 0 \\
$20-30$ & 16 & 1 \\
$31-40$ & 25 & 4 \\
$41-50$ & 22 & 2 \\
$51-60$ & 25 & 9 \\
$>60$ & 11 & 4 \\
\hline
\end{tabular}


The microorganisms cultured from bile and stones are shown in table-2. Gram negative microorganisms were more frequently isolated from the twenty culture positive patients. The commonest gram negative bacteria were Escherichia coli (40\% $\mathrm{n}=8)$, Klebsiella spp (35\% $\mathrm{n}=7)$, Enterobacter spp $(20 \% \mathrm{n}=4)$ and other coliforms $(10 \% \mathrm{n}=2)$.
One Pseudomonas spp was also isolated. Ninety one percent of all isolates were coliforms. Only one gram positive organism was isolated (methicillin sensitive Staphylococcus aureus MSSA). Polymicrobial infection was seen in 15\% $(n=3)$ of the culture positive patients with a maximum of two organisms.

Table 2 - Bacteriology of gallstone disease

\begin{tabular}{|l|c|c|c|}
\hline Organism & $\begin{array}{c}\text { No. isolated from } \\
\text { stones }\end{array}$ & $\begin{array}{c}\text { No. isolated from } \\
\text { bile }\end{array}$ & $\begin{array}{c}\text { No. isolated from } \\
\text { either stones or bile }\end{array}$ \\
\hline Escherichia coli & 7 & 7 & 8 \\
\hline Klebsiella spp & 6 & 6 & 7 \\
\hline Enterobacter spp & 3 & 3 & 4 \\
\hline Other coliforms & 1 & 2 & 2 \\
\hline Pseudomonas spp & 1 & 1 & 1 \\
\hline Staphylococcus aureus & 1 & 0 & 1 \\
\hline
\end{tabular}

Table 3 - Antimicrobial susceptibility of the isolates

\begin{tabular}{|c|c|c|c|c|}
\hline \multicolumn{5}{|l|}{ \% Susceptibility } \\
\hline Antibiotic & 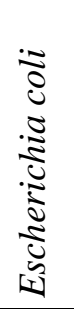 & 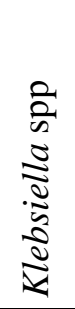 & 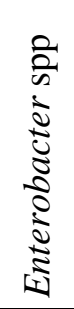 & 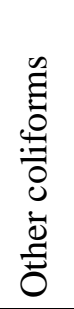 \\
\hline Gentamycin & 100 & 85 & 100 & 100 \\
\hline Amikacin & 100 & 85 & 100 & 100 \\
\hline Co - amoxiclav & 88 & 100 & 50 & 50 \\
\hline Ampicillin & 25 & 0 & 0 & 0 \\
\hline Cefotaxime & 100 & 100 & 100 & 100 \\
\hline Ceftriaxone & 100 & 86 & 100 & 100 \\
\hline Cefuroxime & 75 & 86 & 100 & 100 \\
\hline Ciprofloxacin & 88 & 100 & 100 & 100 \\
\hline Imipenem & 100 & 100 & 100 & 100 \\
\hline Piperacillin & 88 & 57 & 100 & 100 \\
\hline
\end{tabular}

Antibiotic sensitivity test results are shown in table 3. Hundred percent of the coliforms were sensitive to carbapenems (imipenem), 95\% to aminoglycosides (gentamycin and amikacin) and fluoroquinolones (ciprofloxacin), 81\% to coamoxiclav and ureidopenicillins (piperacillin). Third generation cephalosporins (cefotaxime - 100\%, ceftriaxone - 95\%) were more effective against coliforms than the second generation cephalosporin cefuroxime (86\%). Only five percent of the coliforms were sensitive to ampicillin. The Pseudomonas spp was sensitive to aminoglycosides, ciprofloxacin and piperacillin. The MSSA was sensitive to aminoglycosides, cephalosporins, ciprofloxacin and vancomycin.

\section{Discussion and Conclusions}

We have shown a positive culture rate of $20 \%$ in symptomatic gallstone disease. Our results are comparable to previous results, which showed 23$46 \%$ culture positivity in symptomatic gallbladder stones in other parts of the world [15]. Bile is generally sterile but is easily infected when stasis occurs due to obstruction. 
In surgical practice in Sri Lanka cholecystectomy is performed in acute cholecystitis following conservative treatment with antibiotics, making it difficult to obtain specimens. This practice may have reduced our positive rate. Further the inability to culture for anaerobic bacteria, which was a limitation of our study, also could have reduced the positivity rate; however, previous published studies show this to be approximately ten percent [16].

The gram negative enteric organisms formed $91 \%$ of the isolates in which Escherichia coli (40\%), Klebsiella spp (35\%) and, Enterobacter spp (20\%) were the commonest organisms. In previous results gram negative enteric aerobes accounted for $70-78 \%$ of positive bile cultures, in which Escherichia coli and Klebsiella spp were the commonest as seen in our study [16]. There were $15 \%$ of culture positive patients with polymicrobial infection in this study. Previous results show $10-30 \%$ of patients with polymicrobial infection, justifying a selection of a broad spectrum antibiotic [16]. Both bile and gallstones should be cultured for a more accurate picture.

In this study sensitivity for ampicillin was only five percent. However co-amoxiclav (amoxicillin and clavulanic acid) had an efficacy ranging from 50100\%. Aminoglycosides (gentamicin and amikacin), cephalosporins (cefotaxime, ceftriaxone and cefuroxime), ciprofloxacin and imipenem had good coverage ranging from 75$100 \%$.

A combination of a penicillin (usually ampicillin) with an aminoglycoside has been recommended for treatment of acute cholangitits [17]. The same antibiotics along with metronidazole are suggested in Sri Lanka [18]. The administration of ampicillin is insufficient and according to results of our study co-amoxiclav should be used with an aminoglycoside. Considering the possible disadvantages of using aminoglycoside, an alternative treatment against gram negative and gram positive bacteria is cephalosporins.

In conclusion our findings are comparable to other investigations in the world. Enteric bacteria are the commonest bacteria isolated from Sri Lankan patients with symptomatic gallstone disease.
Co-amoxiclav in combination with an aminoglycoside and metronidazole empirically can be used before the results of bacteriological cultures. Cefotaxime or imipenem can be used along with metronidazole as an alternative treatment.

The significant culture positive rate shows the importance of obtaining cultures of bile and gallstones at the time of cholecystectomy so that appropriate antibiotics can be administered and necessary changes made according to the antibiotic sensitivity tests to prevent serious complications like gram negative septicaemia.

\section{Acknowledgements}

We wish to thank University of Sri Jayawardenepura for financial support (grant ASP/6/RE/2004- 4), Surgery Unit, Colombo South Teaching Hospital, Kalubowila.

\section{References}

1. Indar AA, Beckingham IJ. Acute cholecystitis. British Medical Journal 2002; 325: 639-43.

2. Csendes A, Fernandez M, Uribe P. Bacteriology of the gallbladder bile in normal subject. American Journal of Surgery 1975; 129: 629-31.

3. Scott AJ. Bacteria and disease of the biliary tract progress report. Gut 1971; 12: 487-92.

4. Carpenter HA. Bacterial and parasitic cholangitis. Journal of Mayo Clinic Proceedings 1998; 73: 473-8.

5. Kuo CH, Changchien CS, Chen JJ, Tai DI, Chiou SS, Lee CM. Septic acute cholecystitis. Scandinavian Journal of Gastroenterology 1995; 30: 272-5.

6. Jeng KS, Shih SC, Chiang HJ, Chen BF. Secondary biliary cirrhosis. Journal of Archives of Surgery 1989; 124: $1301-5$

7. Fan ST, Lai EC, Mok FP, Choi TK, Wong J. Acute cholangitis secondary to hepatolithiasis. Journal of Archives of Surgery 1991; 126: 1027-31.

8. Fan ST, Lai ECS, Wong J. Hepatic resection for hepatolithiasis. Journal of Archives of Surgery 1993; 128: $1070-4$

9. Lee KT, Sheen PC, Tsai CC, Chen JS, Ker CG. Long term results of one hundred and seven hepatic resection for intrahepatic stones. Journal of Digestive Surgery 1992; 9: 298-302.

10. Boey JH, Way LW. Acute cholangitis. Journal of Annals of Surgery 1980; 191: 264-70. 
11. Elsakr R, Johnson DA, Younes Z, Oldfield EC. Antimicrobial treatment of intra abdominal infections. Journal of Digestive Surgery 1998; 16: 47-60.

12. Ballal M, Jyothi KN, Antony B, Arun C, Prabhu T, Shivananda PG. Bacteriological spectrum of cholecystitis and its antibiogram. Indian Journal of Medical Microbiology 2001;19(4):212-4.

13. Gallstones and laparoscopic cholecystectomy. National Institute of Health Consensus Statement 1992; Sep 14-16: 10(3): 1-28.

14. Bilhartz LE, Horton JD. Gallstone disease and its complications. In: Feldman M, Scharschmidt BF, Sleisenger MH; eds. Sleisenger \& Fordtran’s. Gastrointestinal and liver disease: pathophysiology, diagnosis, management. $6^{\text {th }}$ Ed, Philadelphia, Saunders; 1998: 948-72.
15. Fan ST, Lai EC, Mok FP, Choi TK, Wong J. Acute cholangitis secondary to hepatolithiasis. Journal of Archives of Surgery 1991; 126: 1027-31.

16. Chang WT, Lee KT, Wang SR, Chuang SC, Kuo KK, Chen JS, et al. Bacteriology and antimicrobial susceptibility in biliary tract disease: an audit of 10 years experience. Kaohsiung Journal of Medical Science 2002; 18(5): 221-8.

17. Thompson JE (Jr), Pitt HA, Doty JE, Coleman J, Irving C. Broad spectrum penicillins as an adequate therapy for acute cholangitis. Journal of Surgery Gynaecology and Obstetrics 1990; 171: 275-82.

18. Atukorala SD. Guidelines for rational use of antibiotics. 1991; 1: 30-66. 